\title{
Derivada Topológica em um Problema Inverso de Reconstrução
}

\author{
Antonio A. Novotny, Suelen S. Rocha, \\ Laboratório Nacional de Computação Científica-LNCC \\ 25651-075, Petrópolis, RJ \\ E-mail: novotny@lncc.br, suelen@lncc.br,
}

\begin{abstract}
Resumo: A análise de sensibilidade topológica fornece um desenvolvimento assintótico para um funcional de forma, cujo termo principal é um campo escalar chamado derivada topológica, que mede a sensibilidade de um dado funcional quando uma perturbação singular infinitesimal é introduzida em um ponto do dominio. Dentre outras aplicações bem sucedidas da derivada topológica, destaca-se sua utilização na resolução de certas classes de problemas inversos. Neste trabalho objetiva-se aplicar a derivada topológica de primeira e segunda ordem na resolução de um problema inverso de reconstrução consistindo na detecção de furos a partir de leituras parciais obtidas em parte da fronteira do corpo. Este problema é escrito na forma de uma equação diferencial parcial sobredeterminada. Sendo assim, a ideia básica consiste em reescrever o problema inverso na forma de problema de otimização consistindo na minimização de um funcional de forma que mede a diferença entre o dado lido e o calculado numericamente. A derivada topológica é então utilizada no processo de minimização do funcional de forma adotado conduzindo à resolução do problema inverso em um único passo e sem a necessidade de introduzir qualquer tipo de regularização.
\end{abstract}

Palavras-chave: Análise da sensibilidade topológica, derivada topológica, problema inverso.

\section{Introdução}

Um processo físico é representado por seus dados de entrada, dados de saída e pelos parâmetros do sistema. Em um problema direto tem-se os dados de entrada e os parâmetros do sistema e obtém-se os dados de saída. Já em um problema inverso são buscados os dados de entrada ou os parâmetros do sistema a partir dos dados de saída, em linhas gerais, dado os efeitos de um processo deseja-se obter as causas.

Um problema inverso pode aparecer de duas formas: Problema de reconstrução (dado o sistema de parâmetros e observado o efeito encontrar a causa que corresponde ao efeito) e problema de identificação (dadas as causas e o efeito determinar o sistema de parâmetros que relaciona as causas ao efeito).

Geralmente um problema inverso não possui informação suficiente para descrevê-lo por completo. Podendo faltar informações que garantam a existência, a unicidade ou a dependência contínua dos dados. Um problema é dito bem posto no sentido de Hadamard se cumpre as condições de existência, unicidade e dependência contínua dos dados de entrada. Caso um desses requisitos não seja satisfeito o problema é dito mal-posto. Desta forma, trabalhar com problema inverso frequentemente significa lidar com um problema mal posto.

A derivada topológica foi introduzida por Sokolowski e Zochowski [7] e desde então tem sido utilizada de forma bem sucedida na resolução de diversos tipos de problemas inversos. Em [1] a derivada topológica é usada na detecção e localização de trincas. No artigo [2] a derivada topológica é utilizada na resolução do problema de modelagem eletromagnética que consiste em determinar correntes elétricas e o campo induzido para que um metal líquido assuma um determinado formato. Em [4] um novo método é proposto utilizando derivada topológica para resolução do problema inverso de espalhamento que consiste em determinar as características de um objeto (forma, constituição interna) com base em dados de como ele espalha partículas ou radiação recebida. Já em $[5,6]$ o algoritmo proposto para obtenção da solução do problema de EIT (Electrical Impedance Tomography) é inicializado usando análise de sensibilidade topológica. O problema inverso do potencial também foi tratado com sucesso em [3], onde um novo método foi proposto para resolução de tal problema com o uso da derivada topológica. 
O objetivo deste trabalho é utilizar a derivada topologica na resolução de um problema inverso de reconstrução. Mais precisamente, pretende-se reconstruir a topologia de definição do problema a partir de leituras obtidas na fronteira do mesmo. Primeiramente o problema é transformado em um problema de otimização consistindo na minimização de um funcional de forma que mede a diferença entre o dado lido e o calculado numericamente. A derivada topológica é então utilizada no processo de minimização do funcional de forma adotado conduzindo à resolução do problema inverso.

\section{Derivada Topológica}

Considere $\Omega \subset \mathbb{R}^{d}, d \geq 2$, um domínio aberto e limitado. Seja $\widehat{x}$ um ponto arbitrário de $\Omega$ e $\omega$ um domínio fixo de $\mathbb{R}^{d}$. Introduz-se uma pertubação não suave delimitada em uma região $\omega_{\varepsilon}(\widehat{x})=\widehat{x}+\varepsilon \omega$ com $\overline{\omega_{\varepsilon}} \subset \Omega$. Observe a Figura 1 .

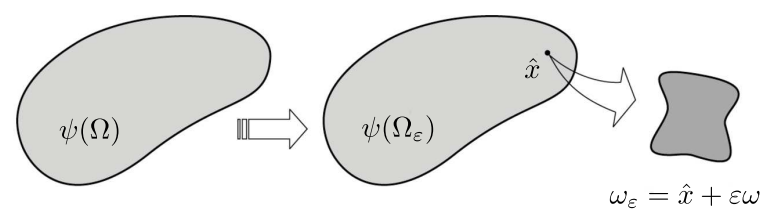

Figura 1: Derivada topológica

Assuma que um dado funcional de forma $\psi\left(\Omega_{\varepsilon}(\widehat{x})\right)$ associado ao domínio topologicamente perturbado $\Omega_{\varepsilon}=\Omega \backslash \overline{w_{\varepsilon}(\hat{x})}$ admite a seguinte expansão assintótica

$$
\psi\left(\Omega_{\varepsilon}(\widehat{x})\right)=\psi(\Omega)+f(\varepsilon) D_{T} \psi(\widehat{x})+o(f(\varepsilon))
$$

onde $\psi(\Omega)$ é o funcional de forma associado ao domínio não perturbado $\Omega, f(\varepsilon)$ é uma função regular positiva e $o(f(\varepsilon))$ é tal que $\frac{o(f(\varepsilon))}{f(\varepsilon)} \rightarrow 0$ quando $\varepsilon \rightarrow 0$. A função $\widehat{x} \mapsto D_{T} \psi(\widehat{x})$ é chamada de derivada topológica de $\psi$ em $\widehat{x}$. Rearranjando a equação (1) e passando ao limite obtemos a seguinte expressão para a derivada topológica:

$$
D_{T} \psi(\widehat{x}):=\lim _{\varepsilon \rightarrow 0} \frac{\psi\left(\Omega_{\varepsilon}(\widehat{x})\right)-\psi(\Omega)}{f(\varepsilon)} .
$$

Considere agora a seguinte expansão

$$
\psi\left(\Omega_{\varepsilon}(\widehat{x})\right)=\psi(\Omega)+f(\varepsilon) D_{T} \psi(\widehat{x})+f_{2}(\varepsilon) D_{T}^{2} \psi(\widehat{x})+R\left(f_{2}(\varepsilon)\right)
$$

onde $D_{T} \psi$ e $D_{T}^{2} \psi$ são as derivadas topológicas do funcional de forma $\psi$ de primeira e segunda ordem respectivamente.

A função $f_{2}(\varepsilon)$ é positiva, monótona e $f_{2}(\varepsilon) \rightarrow 0$ quando $\varepsilon \rightarrow 0$. Ademais,

$$
\lim _{\varepsilon \rightarrow 0} \frac{f_{2}(\varepsilon)}{f(\varepsilon)}=0
$$

Rearranjando a equação (3) e passando ao limite obtemos a seguinte expressão para a derivada topológica de segunda ordem:

$$
D_{T}^{2} \psi(\widehat{x}):=\lim _{\varepsilon \rightarrow 0} \frac{\psi\left(\Omega_{\varepsilon}(\widehat{x})\right)-\psi(\Omega)-f(\varepsilon) D_{T} \psi(\widehat{x})}{f_{2}(\varepsilon)} .
$$

\section{Formulação do Problema}

Considere um domínio $\Omega \subset \mathbb{R}^{2}$ aberto e limitado com fronteira suave. Este domínio possui um furo $\omega_{0}$ e uma fonte distribuída $b$ em seu interior. Como mostrado na Figura 2(a). Além disso, o domínio $\Omega$ possui anomalias (furos) não conhecidas em seu interior que é denotada por $\omega^{*}$. Observe a Figura 2(b). 


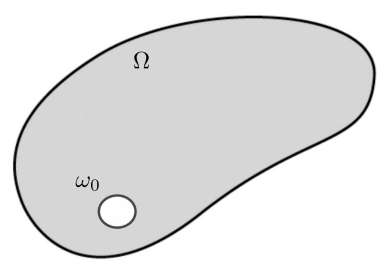

(a) Domínio sem anomalias

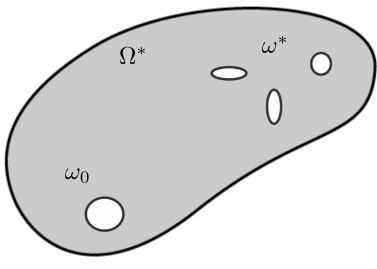

(b) Domínio com anomalias

Figura 2: Domínio

Considere o campo $u$ conhecido na fronteira de $\Omega$ e assuma $b$ constante. O objetivo é reconstruir $\Omega^{*}$. Logo, nosso problema consiste em determinar $\omega^{*}$ tal que

$$
\left\{\begin{array}{l}
-\Delta u=b \text { em } \Omega^{*}=\Omega \backslash \overline{\omega^{*}} \\
u=0 \text { sobre } \Gamma_{0}=\partial \omega_{0} \\
u=0 \text { sobre } \partial \omega^{*} \\
u=u^{*} \text { sobre } \Gamma_{m} \\
-\partial_{n} u=q^{*} \text { sobre } \Gamma_{m}
\end{array}\right.
$$

onde $\Gamma_{m}$ denota a fronteira exterior de $\Omega$ e $\Gamma_{0}$ denota a fronteira de $\omega_{0}$. Temos um problema sobredeterminado já que dados de Dirichlet e Neumann são prescritos sobre a fronteira $\Gamma_{m}$ simultaneamente. Pode-se considerar os seguintes problemas inversos:

- Dada a excitação de Dirichlet $u^{*}$ sobre $\Gamma_{m}$ podemos observar $q^{*}$ sobre $\Gamma_{m}$ para encontrar $\omega^{*}$.

- Dada a excitação de Neumann $q^{*}$ sobre $\Gamma_{m}$ podemos observar $u^{*}$ sobre $\Gamma_{m}$ para obter $\omega^{*}$.

Escolhemos a primeira maneira de tratar o problema.

\subsection{Reformulação do Problema}

O problema considerado é mal posto e está escrito na forma de uma equação diferencial parcial sobredeterminada. Nesta seção reescreve-se o problema inverso apresentado na seção anterior na forma de um problema de otimização. Considere o seguinte funcional de forma:

$$
\psi(\Omega)=\int_{\Gamma_{m}}\left(u_{0}-u^{*}\right)^{2},
$$

onde $u_{0}$ é solução do seguinte problema:

$$
\left\{\begin{array}{l}
-\Delta u_{0}=b \text { em } \Omega \\
u_{0}=0 \text { sobre } \Gamma_{0} \\
-\partial_{n} u_{0}=q^{*} \text { sobre } \Gamma_{m}
\end{array} .\right.
$$

Proposição 1 Seja $u_{0}$ solução do problema variacional (6). Então, se $\Omega=\Omega^{*}$, onde $\Omega^{*}$ é uma solução do problema inverso (4), tem-se que $u_{0}=u^{*}$ sobre $\Gamma_{m}$ e portanto o funcional (5) atinge um mínimo sobre $\Gamma_{m}$.

A Proposição 1 garante que sobre a solução do problema inverso o funcional (5) atinge o seu valor mínimo sobre $\Gamma_{m}$, ou seja, $u_{0}=u^{*}$ sobre $\Gamma_{m}$. Assim, encontrar o mínimo do funcional (5) é equivalente a resolver o problema (4). 
Considere agora o funcional de forma associado ao domínio perturbado $\Omega_{\varepsilon}=\Omega \backslash \overline{B_{\varepsilon}(\hat{x})}$ dado por

$$
\psi\left(\Omega_{\varepsilon}\right)=\int_{\Gamma_{m}}\left(u_{\varepsilon}-u^{*}\right)^{2}
$$

Com $u_{\varepsilon}$ solução do seguinte problema perturbado

$$
\left\{\begin{array}{l}
\text { Encontre } u_{\varepsilon}, \text { tal que } \\
-\Delta u_{\varepsilon}=b \text { em } \Omega_{\varepsilon} \\
u_{\varepsilon}=0 \text { sobre } \Gamma_{0} \\
-\partial_{n} u_{\varepsilon}^{N}=q^{*} \text { sobre } \Gamma_{m} \\
u_{\varepsilon}=0 \text { sobre } \partial B_{\varepsilon} .
\end{array}\right.
$$

onde $B_{\varepsilon}(\hat{x})$ denota a bola de centro em $\hat{x}$ e raio $\varepsilon$.

\section{Cálculo da Derivada Topológica}

Afim de identificar $f(\varepsilon), f_{2}(\varepsilon)$, calcular $D_{T} \psi(\hat{x})$ e $D_{T}^{2} \psi(\hat{x})$ precisamos conhecer o comportamento da função $u_{\varepsilon}$ com respeito a $\varepsilon$ na vizinhança de $B_{\varepsilon}$. Uma vez conhecido seu comportamento podemos identificar as funções $f(\varepsilon), f_{2}(\varepsilon)$ com a finalidade de obter a fórmula final para as derivadas topológica de primeira e segunda ordem. Sendo assim, é necessário realizar a análise assintótica de $u_{\varepsilon}$ com respeito a $\varepsilon$.

A seguinte expansão para do problema (8) é proposta

$$
u_{\varepsilon}(x)=u_{0}(x)+\varphi_{\varepsilon}(x)+\widetilde{u}_{\varepsilon}(x),
$$

onde $\varphi_{\varepsilon}(x)=\alpha(\varepsilon) u_{0}(\widehat{x}) G(x)$.

A função $G(x)$ é solução do seguinte problema:

$$
\left\{\begin{array}{l}
-\Delta G=\delta(x-\widehat{x}) \quad \text { em } \Omega \\
\partial_{n} G=0 \text { sobre } \Gamma_{m} \\
G=0 \text { sobre } \Gamma_{0}
\end{array} .\right.
$$

Note que $G(x)=G(x ; \hat{x})$. Por meio da solução fundamental para o Laplaciano admite na vizinhança de $\widehat{x} \in \Omega$ a seguinte representação

$$
G(x)=-\left(\frac{1}{2 \pi} \ln \|x-\widehat{x}\|+g(x)\right),
$$

onde $g(x)$ é solução do seguinte problema auxiliar

$$
\left\{\begin{array}{l}
-\Delta g=0 \quad \text { em } \Omega \\
\partial_{n} g=-\frac{1}{2 \pi} \frac{x-\widehat{x}}{\|x-\widehat{x}\|^{2}} \cdot n \quad \text { sobre } \Gamma_{m} \\
g=-\frac{1}{2 \pi} \ln \|x-\widehat{x}\| \quad \text { sobre } \Gamma_{0}
\end{array} .\right.
$$

Da mesma forma $g(x)=g(x ; \hat{x})$. Expandindo $u_{0}$ em série de Taylor na expansão $(9)$ obtemos

$$
u_{\varepsilon}(x)=u_{0}(\widehat{x})+\nabla u_{0}(y)(x-\widehat{x})+\varphi_{\varepsilon}(x)+\widetilde{u}_{\varepsilon}(x),
$$

onde $y$ é um ponto entre $x$ e $\widehat{x}$. 
Lembrando que $\left.u_{\varepsilon}\right|_{\partial B_{\varepsilon}}=0$ e usando a regularidade da função $g$ na vizinhança de $\widehat{x}$ a expansão para $u_{\varepsilon}$ sobre $\partial B_{\varepsilon}$ é dada por

$$
u_{0}(\widehat{x})+\varepsilon \nabla u_{0}(y) \cdot n-\alpha(\varepsilon) u_{0}(\widehat{x})\left(\frac{1}{2 \pi} \ln \varepsilon+g(\widehat{x})+\varepsilon \nabla g(z) \cdot n\right)+\widetilde{u}_{\varepsilon}(x)=0,
$$

onde $z$ é um ponto entre $x$ e $\widehat{x}$.

A função $\widetilde{u}_{\varepsilon}$ é contruída de forma que a discrepância introduzida pelos termos com ordem em $\varepsilon$ seja compensada. Assim,

$$
\widetilde{u}_{\varepsilon}(x)=\varepsilon\left(\alpha(\varepsilon) u_{0}(\widehat{x}) \nabla g(z) \cdot n-\nabla u_{0}(y) \cdot n\right) \quad \text { sobre } \partial B_{\varepsilon} .
$$

A expansão (10) se reduz a

$$
u_{0}(\widehat{x})-\alpha(\varepsilon) u_{0}(\widehat{x})\left(\frac{1}{2 \pi} \ln \varepsilon+g(\widehat{x})\right)=0 \quad \text { sobre } \partial B_{\varepsilon} .
$$

Donde

$$
\alpha(\varepsilon)=\frac{2 \pi}{\ln (\varepsilon)+2 \pi g(\hat{x})} .
$$

Constroi-se a função $\widetilde{u}_{\varepsilon}$ de tal forma que a diferença introduzida pelos termos da expansão para $u_{\varepsilon}$ seja compensada, ou seja, $\widetilde{u}_{\varepsilon}$ deve satisfazer

$$
\left\{\begin{array}{l}
\Delta \widetilde{u}_{\varepsilon}=0 \quad \text { em } \Omega_{\varepsilon} \\
\partial_{n} \widetilde{u}_{\varepsilon}=0 \quad \text { sobre } \Gamma_{m} \\
\widetilde{u}_{\varepsilon}=\varepsilon\left(\alpha^{N}(\varepsilon) u_{0}(\widehat{x}) \nabla g(z) \cdot n-\nabla u_{0}(y) \cdot n\right) \quad \text { sobre } \partial B_{\varepsilon} \\
\widetilde{u}_{\varepsilon}=0 \quad \text { sobre } \Gamma_{0}
\end{array}\right.
$$

onde $y$ e $z$ são pontos entre $x$ e $\widehat{x}$. Assumindo regularidade suficiente temos que $\tilde{u}_{\varepsilon}$ é de ordem $O(\varepsilon)$. A expansão para $u_{\varepsilon}$ é dada por:

$$
u_{\varepsilon}(x)=u_{0}(x)-\alpha(\varepsilon) u_{0}(\widehat{x})\left(\frac{1}{2 \pi} \log \|x-\widehat{x}\|+g(\widehat{x})\right)+O(\varepsilon) .
$$

Considere a expansão (9) proposta:

$$
u_{\varepsilon}(x)=u_{0}(x)+\varphi_{\varepsilon}(x)+\widetilde{u}_{\varepsilon}(x)
$$

onde $\varphi_{\varepsilon}(x)=\alpha(\varepsilon) u_{0}(\hat{x}) G(x)$. Como $\widetilde{u}_{\varepsilon}(x)$ é de ordem $O(\varepsilon)$ tem-se que

$$
u_{\varepsilon}(x)=u_{0}(x)+\varphi_{\varepsilon}(x)+O(\varepsilon) .
$$

Substituindo (14) em (7) tem-se

$$
\psi\left(\Omega_{\varepsilon}\right)=\psi(\Omega)+2 \alpha(\varepsilon) u_{0}(\hat{x}) \int_{\Gamma_{m}}\left(u_{0}-u^{*}\right) G+\alpha(\varepsilon)^{2} u_{0}(\hat{x})^{2} \int_{\Gamma_{m}} G^{2}+O(\varepsilon) .
$$

Considere $f(\varepsilon)=\alpha(\varepsilon)$ e $f_{2}(\varepsilon)=\alpha(\varepsilon)^{2}$. Comparando as expansões (15) e (3) tem-se que

$$
D_{T} \psi(\hat{x})=2 u_{0}(\hat{x}) \int_{\Gamma_{m}}\left(u_{0}-u^{*}\right) G \quad \text { e } \quad D_{T}^{2} \psi(\hat{x})=u_{0}(\hat{x})^{2} \int_{\Gamma_{m}} G^{2} .
$$

A expansão assintótica indica o sinal que a derivada topológica deve possuir para que o funcional de forma $\psi$ diminua à medida que o domínio $\Omega$ é perturbado. Logo, como as funções $f(\varepsilon)$ e $f_{2}(\varepsilon)$ são positivas afim de minimizar o funcional de forma adotado deve-se buscar as regiões onde a derivada topológica torna-se negativa.

Agora, introduzimos a quantidade

$$
\Psi(\alpha(\varepsilon))=2 \alpha(\varepsilon) u_{0}(\hat{x}) \int_{\Gamma_{m}}\left(u_{0}-u^{*}\right) G+\alpha(\varepsilon)^{2} u_{0}(\hat{x})^{2} \int_{\Gamma_{m}} G^{2} .
$$


Quer-se minimizar a equação acima em relação a $\alpha(\varepsilon)$. Para tal deriva-se (16) em relação a $\alpha(\varepsilon)$ e igualá-se a zero obtendo o seguinte valor

$$
\alpha^{\star}(\hat{x})=-\frac{\int_{\Gamma_{m}}\left(u_{0}(x)-u^{*}(x)\right) G(x ; \hat{x})}{u_{0}(\hat{x}) \int_{\Gamma_{m}} G(x ; \hat{x})^{2}} .
$$

De (11) e (17) segue que

$$
\varepsilon^{\star}(\hat{x})=\mathrm{e}^{2 \pi\left(\frac{1}{\alpha^{\star}(\hat{x})}-g(\hat{x})\right)} .
$$

Substituindo o valor ótimo $\alpha^{\star}(\hat{x})$ em (16) obtém-se

$$
\Psi\left(\alpha^{\star}(\hat{x})\right)=\alpha^{\star}(\hat{x}) u_{0}(\hat{x}) \int_{\Gamma_{m}}\left(u_{0}(x)-u^{*}(x)\right) G(x ; \hat{x}) .
$$

Logo, as localizações ótimas dos furos $\hat{x}^{\star}$ são obtidas simplesmente buscando-se os pontos $\hat{x}$ que fornecem os menores valores para $\Psi\left(\alpha^{\star}(\hat{x})\right)$.

\section{Resultados Numéricos}

Considere o domínio $\Omega$ dado por uma circunferência de centro em $(0,0)$ e raio 1 que possui um furo de centro em $(-0.5,0)$ e raio 0.3 (observe a Figura $3(\mathrm{a})$ ). Deseja-se obter a localização e tamanho de alguma anomalia (furo) adicional no interior do domínio $\Omega$. No experimento apresentado quer-se reconstruir $\Omega^{*}$ representado na Figura 3(b), onde tem-se uma anomalia localizada no ponto $(0.4,0.4)$ com raio 0.2. Para tal primeiramente realizamos duas medidas na fronteira de $\Omega^{*}$. Na primeira impoe-se um fluxo $q_{1}^{*}=x$, na segunda um fluxo $q_{2}^{*}=y$ e são lidos os campos $u_{1}^{*}$ e $u_{2}^{*}$ respectivamente.

Na Figura 4(a) nota-se que a derivada topológica de primeira ordem não fornece informação suficiente para detectarmos a anomalia, uma vez que ela atinge os valores mais críticos na fronteira. Desta forma, é proposta a utilização da expansão truncada (16) que possui um termo a mais. A expansão (16) é uma forma quadrática em $\alpha(\varepsilon)$ e, portanto, possui um ponto de mínimo global. Calcula-se inicialmente o mínimo de (16) em relação a $\alpha(\varepsilon)$ obtendo o valor $\alpha^{\star}(\hat{x})$ dado por (17).

Daí, substituindo $\alpha^{\star}(\hat{x})$ em (16) tem-se (19) donde a localização ótima da anomalia $x^{\star}$ é obtida buscando o ponto que fornece o menor valor para $\Psi\left(\alpha^{\star}(\hat{x})\right)$. E o tamanho ótimo da anomalia dado pela substituição de (17) em (11) obtendo o valor $\varepsilon^{\star}$ dado em (18). A solução é dada então pelo par $\left(x^{\star}, \varepsilon^{\star}\right)$.

No experimento obtemos a localização exata da anomalia $x^{\star}=(0.4,0.4)$ com um raio um pouco superestimado dado por $\varepsilon^{\star}=0,225$ que representa um erro relativo de $12.5 \%$. Considerando a derivada topológica de segunda ordem observa-se que as discrepâncias são corrigidas, de forma que funcional associado ao problema perturbado atinge seu mínimo exatamente no centro das anomalias. Vale salientar que o resultado apresentado foi obtido em apenas uma iteração. Observe a Figura 4(b).

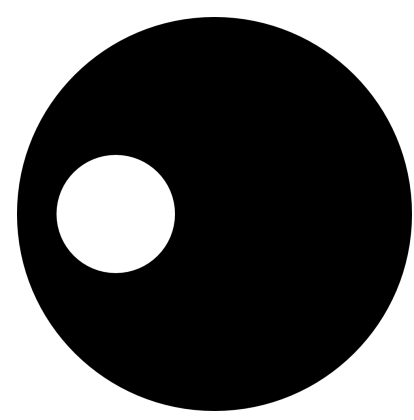

(a) $\Omega$

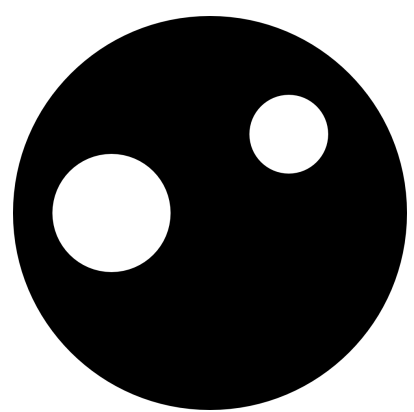

(b) $\Omega^{*}$

Figura 3: Domínio inicial (a) e Domínio a ser reconstruído (b). 


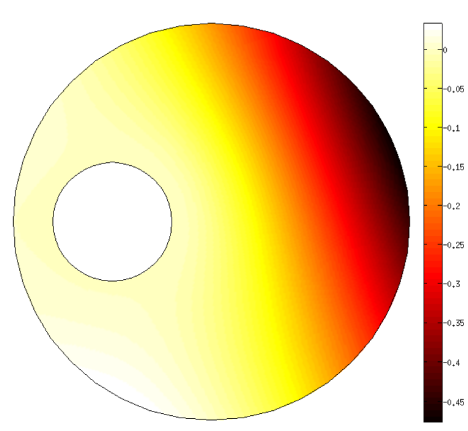

(a) $D_{T} \psi$

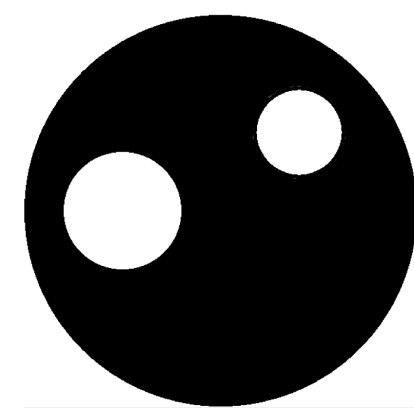

(b) $x^{\star} \mathrm{e} \varepsilon^{\star}$

Figura 4: Resultados obtidos utilizando a derivada de primeira ordem $(a)$ e as derivadas de primeira e segunda ordem $(b)$.

\section{Conclusão}

O intuito do trabalho foi resolver um problema inverso de reconstrução consistindo na detecção de furos a partir de leituras parciais obtidas em parte da fronteira do corpo. Este problema é escrito na forma de uma equação diferencial parcial sobredeterminada. O problema inverso foi reescrito na forma de problema de otimização consistindo na minimização de um funcional de forma que mede a diferença entre o dado lido e o calculado numericamente. Foi apresentado o cálculo das derivadas topológica de primeira e segunda ordem para o funcional de forma adotado. A derivada topológica de primeira ordem fornece a direção de descida do funcional de forma e, portanto, faz sentido sua utilização no processo de minimização. Porém, com a utilização apenas da derivada topológica de primeira ordem não foi possível apresentar uma solução para o problema. Assim, foi introduzido um termo a mais na expansão do funcional de forma associado ao domínio não perturbado, a saber a derivada topológica de segunda ordem que corrigiu as discrepâncias apresentadas pela derivada topológica de primeira ordem. Após o truncamento da expansão, o problema resultante é dado por dois problemas triviais de otimização, onde o primeiro fornece o tamanho ótimo do furo, enquanto que o segundo fornece a sua localização ótima. O método resultante é não iterativo e conduz a uma boa aproximação da solução em um único passo e não necessita de nenhuma regularização. Existe a possibilidade de extensão para vários furos, o que permitirá reconstruir um domíno com várias anomalias simultaneamente em um único passo.

\section{Referências}

[1] Amstutz, S.; Horchani, I. Masmoudi; M., Crack detection by the topological gradient method. Control and Cybernetics 34(1), 81-101 (2005).

[2] Canelas, A.; Novotny, A.A.; Roche, J.R., A new method for inverse electromagnetic casting problems based on the topological derivative, Journal of Computational Physics 230, 3570-3588 (2011).

[3] Canelas, A; Laurain, A.; Novotny A. A., A new reconstruction method for the inverse potential problem, Journal of Computational Physics 268, 417-431 (2014).

[4] Feijóo, G.R., A new method in inverse scattering based on the topological derivative. Inverse Problems 20(6), 1819-1840 (2004).

[5] Hintermüller, M., Laurain, A., Electrical impedance tomography: from topology to shape. Control and Cybernetics 37(4), 913-933 (2008).

[6] Hintermüller, M., Laurain, A., Novotny, A.A., Second-order topological expansion for electrical impedance tomography. Advances in Computational Mathematics 36(2), 235-265 (2012).

[7] Sokolowski, J.; Zochowski,A., On the topological derivative in shape optimization. SIAM Journal on Control and Optimization 37(4), 1251-1272 (1999) 